This is a self-archived - parallel published version of this article in the publication archive of the University of Vaasa. It might differ from the original.

\title{
An optimal approach for load-frequency control of islanded microgrids based on nonlinear model
}

Author(s): Jamshidi, Fatemeh; Salehizadeh, Mohammad Reza; Gholami, Fatemeh; Shafie-khah, Miadreza

Title: $\quad$ An optimal approach for load-frequency control of islanded microgrids based on nonlinear model

Year: $\quad 2020$

Version: Final draft (post print, aam. accepted manuscript)

Copyright (C)2020 Springer Nature Switzerland AG. This is a post-peer-review, precopyedit version of an article published in Amini, M. (ed). Optimization, learning, and control for interdependent complex networks. Advances in intelligent systems and computing, vol. 1123. Springer, Cham. The final authenticated version is available online at: https://doi.org/10.1007/ 978-3-030-34094-0_11.

\section{Please cite the original version:}

Jamshidi, F., Salehizadeh, M.R., Gholami, F., \& Shafie-khah, M., (2020) An optimal approach for load-frequency control of islanded microgrids based on nonlinear model. In: Amini, M. (ed). Optimization, learning, and control for interdependent complex networks (pp. 257-275). Advances in intelligent systems and computing, vol. 1123. Springer, Cham. https://doi.org/10.1007/978-3-030-34094-0_11 


\title{
An Optimal Approach for Load-Frequency Control of Islanded Microgrids Based on Nonlinear Model
}

\author{
Fatemeh Jamshidi' ${ }^{1}$, Mohammad Reza Salehizadeh ${ }^{2,}{ }^{,}$, Fatemeh Gholami², Miadreza \\ Shafie-khah ${ }^{3}$ \\ 1 Department of Electrical Engineering, Faculty of Engineering, Fasa University, Fasa, Iran. \\ 2 Department of Electrical Engineering, Islamic Azad University, Marvdasht Branch, Marvdasht, Iran. \\ ${ }^{3}$ School of Technology and Innovations, University of Vaasa, 65200 Vaasa, Finland. \\ ${ }^{*}$ Corresponding author info: Department of Electrical Engineering, Islamic Azad University, Marvdasht Branch, \\ Marvdasht, Iran; salehizadeh@miau.ac.ir
}

\author{
KEYWORDS: \\ Microgrid, \\ Intelligent Control, \\ Improved differential \\ evolution (DE), \\ Fuzzy Fractional Order PID \\ Controller
}

\begin{abstract}
Due to the increased environmental and economic challenges, in recent years, renewable based distribution generation has been developed. More penetrations from the side of consumers caused a new concept called microgrids which are able to stand with or without connection to the bulk power system. Control of microgrids in islanded mode is very crucial for decreasing the amplitude of frequency deviations as well as damping speed. This chapter aims to propose an Optimal Combination of FOPD and Fuzzy Pre-compensated FOPI Approach for load-frequency control of microgrids in islanded mode. The optimization parameter of the control scheme is designed by the differential evolution (DE) algorithm which has been improved by a fuzzy approach. In the optimization, control effort is considered as a constraint. Due to the robustness and flexibility of the proposed method, the simulation results have been improved substantially. Robust performance of the proposed control method is examined through sensitivity analysis.
\end{abstract}

\section{INTRODUCTION}

After the era of post-restructuring, bulk-power systems have been faced with different challenges such as, but not limited to the lack of available capacity due to transmission congestion, environmental concerns, as well as new problems related to energy market. Environmental concerns, high cost of installing new power plants and barriers in front of transmission expansion planning motivates proliferation of distributed generation units in power industry. Thanks to the development of new small-scale generation technologies, newborn stand-alone grid, called microgrid, have been developed. Microgrids are small power systems that work in low voltage and consist of conventional and renewable power generations, controllable and uncontrollable loads. Microgrids could be operated in two modes: on-grid and off-grid (with or without connection to the upper-level networks). One of the most advantages of a microgrid is the islanding capability and independent operation. The advantage of islanding of a microgrid is increasing the reliability of the consumers connected to the microgrid. In both operation mode reacting to the rapid changes in power consumption is very necessary. In the on-grid mode, frequency and power 
of microgrid depend on the main grid. However, in the islanding mode, frequency and voltage of microgrid oscillate and independent control is required. By disconnecting from the main grid, operation and duties of the microgrid's resources change. The new duties include voltage and frequency control, power- sharing between the resources and appropriate response to the variation of load and the other disturbances. The power system consists of different components which transmit electrical energy in large-scale. In the case of variation in loads or generated power of the resources, frequency variations. A lack of sufficient attention to this problem might cause frequency instability. If generated power is less than the consumed power, frequency decreases. The larger is the system, load variation affects less on the frequency of the systems. In this regard, effective control approaches play a very important role to maintain frequency within its acceptable range with less oscillation. The goal of designing loadfrequency controller is to decrease frequency oscillation and damping of the disturbance in frequency from the viewpoint of domain or time in the normal operation and the case of disturbance [1-5].

Various literature addressed different load-frequency control in the microgrid. The most commonly used control approach for load-frequency control is the proportional-integral-derivative (PID) controller [6]. In [7-8], PI or PID controllers have been used. Optimal tuning of PID parameters is very important for getting the best dynamic performance. To this purpose, in the literature, different methods such as Harmonic Search (HS) [9]. Social-Spider Optimizer (SSO) [10], and Particle Swarm Optimization (PSO) [11] have been used in load-frequency control of microgrid. On the other hand, the performance of the classic PID controller is deteriorated while the operating conditions change. To tackle this challenge, the fuzzy approach is an effective solution to determine the PID parameters. The performance of the fuzzy approach depends on its membership. In [12], fuzzy control has been used and its coefficients have been optimized using PSO, simultaneously with load change. In [13], a fuzzy PSO-based controller has been adopted for the sake of frequency control. In the optimization approach frequency deviations, without paying attention to the control effort, is considered. In [14], an adaptive fuzzy P-PID controller is applied whose parameters have been optimized using an objective function including frequency deviations and eigenvalues. In addition to the PID controller, more advanced control schemes are used for the sake of loadfrequency control. In [15], model predictive control (MPC) has been used. In [16], multiple prediction control has been deployed for load frequency control in the microgrid. In [17], a model-predictive coordinated control of wind turbine blades and the hybrid electric vehicle has been used for reducing power and frequency oscillation. In [18], in the proposed load-frequency control approach, wind turbine blades and PHEVs are controlled using MPC. In the above-mentioned control approaches, a linear model of the microgrid has been used for load-frequency control. Although the small-signal model can be used to study the dynamic behavior of microgrids, there are several phenomena in microgrids whose nonlinear nature must be taken into account in load-frequency control. Nonlinear factors include non-linearity of the output power of some distributed generation sources (such as wind turbine and solar cell) in terms of inputs, limitations of power conversion rates in energy sources and storage, limitation of energy capacity in storage systems and saturation phenomena [19-21].In power system literature, numerous references can be found in which nonlinear phenomena (such as time delay of communication systems, limitations of power rate variations, and saturation phenomena) in load-frequency control have been considered [22-24]; A nonlinear model- based load-frequency control has been proposed in some researches. In [25], the robust (Hळ) method has been used and the limitation of power reserve rate variations has been considered. In [26-29], PI or PID controllers- whose parameters are determined in different ways- are used to control the load-frequency in a nonlinear model-based microgrid. In [26] Fractional- Order PID (FOPID) controller, in [27], Fractional order fuzzy control based PID (FOFCPID), in [28-29], type II fuzzy system have been used. The nonlinear limitations of the generators in [26-27] are considered. Different control strategies have been proposed to improve system performance despite uncertainties. These include optimal control in [30], sliding mode control in [31], intelligent control in [32] and robust control in [25]. PID control is the most commonly used commercial controller with three design parameters: proportional, integral, and derivative coefficients. On the other hand, fractional calculus has received more attention in recent years. In recent years, fractional calculus has been used for system modeling and controller design. Fractional order PID (FOPID) is the most famous fractional order controller. Some efforts have been made to apply FOPID for load-frequency control purposes. As an instant, in [33], a combination of FOPID and ICA optimization approach is used. Frequency deviations is considered in the objective function. The approach is very prospective for load-frequency control. However, control effort is not considered in the proposed unconstraint objective function. Hence, the application of an improved FOPID control approach seems very necessary.

For the above-mentioned purpose, in this chapter, the optimal combination of FOPD and fuzzy precompensated FOPI Approach is proposed and applied for load-frequency control. Here, nonlinear phenomena have been added to the linear model of microprocessor components in dynamic network modeling, including saturation 
and rate limiting. The flexible and robust proposed control scheme is able to improve frequency deviations. Note that optimizing the parameters of this control approach is more complicated in comparison with the conventional fuzzy PID controller. For this purpose, a meta-heuristic approach, differential evolution (DE) algorithm which has been improved by the fuzzy approach, is applied. The purpose of control is to minimize frequency deviations with limited control effort. Microgrid simulation is done in MATLAB environment. The dynamic model of various disturbances, including load changes, changes in wind speed and changes in sunlight, are considered. Results with PID controller whose coefficients are optimized by fuzzy differential evolution algorithm, fractional order PID controller whose coefficients are optimized by fuzzy differential evolution algorithm and FOPD + FFOPI controller whose coefficients are compared. The proposed controller is observed to perform better in damping of oscillations. Also, with the same number of iterations and with the same initial population, better results have been obtained by fuzzy differential evolution algorithm. The simulations have been carried out considering and without considering the uncertainty of the parameters of the dynamic grid model. Considering the considerable variations of the model parameters, the robustness of the proposed control scheme is confirmed.

The chapter is organized as follows: after providing an introduction in section 1, the dynamic model of the microgrid is provided in section 2 . The proposed control method is presented in section 3 . Simulation results are presented in section 4 . Finally, section 5 concludes the chapter.

\author{
Nomenclature \\ $K_{\text {BESS }} \quad$ BESS gain \\ $G_{B E S S}(s) \quad$ BESS Linear Transfer Function \\ $T_{B E S S} \quad$ BESS time constant \\ $u \quad$ Control effort \\ $G_{D E G}(s) \quad$ DEG Linear Transfer Function \\ $T_{T} \quad$ DEG time constant \\ $K_{D} \quad$ Derivative gain of FOPD \\ $P_{D E G} \quad$ Electrical power of DEG \\ $G_{F C}(s) \quad$ FC Linear Transfer Function \\ $T_{I N} \quad$ FC time constant \\ $T_{I C} \quad$ FC time constant \\ $T_{G} \quad$ FC time constant \\ $T_{F C} \quad$ FC time constant \\ $K_{\text {FESS }} \quad$ FESS gain \\ $G_{F E S S}(s) \quad$ FESS Linear Transfer Function \\ $T_{F E S S} \quad$ FESS time constant \\ $K_{p 2} \quad$ FOPI proportional gain \\ $\beta \quad$ Fractional order of Integral
}




\begin{tabular}{|c|c|}
\hline$\alpha$ & Fractional order of the derivative \\
\hline$K_{I}$ & Integral gain of FOPI \\
\hline$P_{L}$ & Load power \\
\hline$\Delta f$ & Microgrid frequency deviations \\
\hline$P_{B E S S}$ & Output electrical power of BESS \\
\hline$P_{F C}$ & Output electrical power of FC \\
\hline$P_{\text {FESS }}$ & Output electrical power of FESS \\
\hline$P_{P V}$ & Output electrical power of PV \\
\hline$K_{p 1}$ & Proportional gain of FOPD \\
\hline$G_{P V}(s)$ & PV Linear Transfer Function \\
\hline$P_{\text {sol }}$ & The solar heat power (light intensity) \\
\hline$G_{\text {load }}(s)$ & Transfer function of the model of load disturbance \\
\hline$G_{s o l}(s)$ & Transfer function of solar power generation model \\
\hline$G_{\text {wind }}(s)$ & Transfer function of wind power generation model \\
\hline$P_{W}$ & Wind mechanical power \\
\hline$P_{W T G}$ & WTG electrical power \\
\hline$K_{W}$ & WTG Gain \\
\hline$G_{W T G}(s)$ & WTG Linear Transfer Function \\
\hline$T_{W}$ & WTG time constant \\
\hline
\end{tabular}

\section{DyNAMic MOdel OF Microgrid}

A microgrid consists of DGs which electrifies local loads in two operational modes, connected to the network and islanded. Because of the intermittent nature of small-scale renewable resources that are connected to the microgrids, energy storage is required to help the system to stand in a stable way. In this chapter, information related to the examined microgrid is obtained from [12]. This microgrid includes diesel engine generators (DEG), Photovoltaic (PV), wind turbine generator (WTG), fuel cells (FC), battery energy storage system (BESS), and flywheel energy storage system (FESS). Table 1 shows the transfer functions of the mentioned components.

It is mentioned that, in Table $1, \mathrm{~K}$ and $\mathrm{T}$ represent gain and time constant, respectively. Figure 1 shows the nonlinear model of the test microgrid which is to be controlled. In the practical applications, the generated power of FESS, BESS, DEG, and FC and their derivatives are limited. In this regard, power generation constraint (GC) and power generation rate constraint (GRC) blocks are considered in the nonlinear model of the test microgrid. For input $P$, their output, $q$, is as follows: 


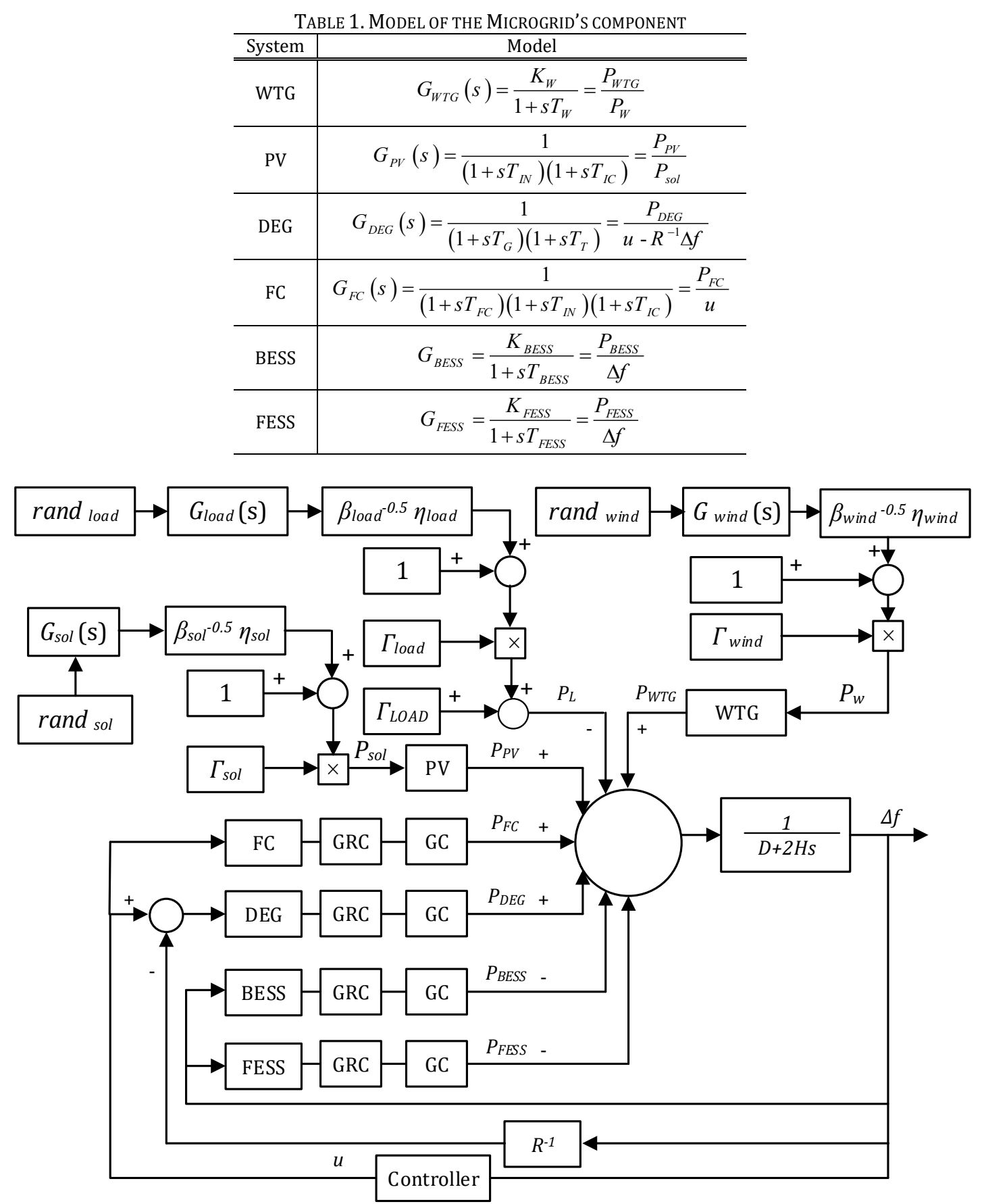

Figure 1: Nonlinear Model of the Test Microgrid

$q=\left\{\begin{array}{cc}P_{\min } & P<P_{\min } \\ P & P_{\min } \leq P \leq P_{\max } \\ P_{\max } & P>P_{\max }\end{array}\right.$

The input of the GRC is a derivative of generated power, and the input of the GC is generated power.

\section{THE PROPOSED INTELLIGENT CONTROL METHOD}

Figure 2 shows the proposed control approach used in this chapter. It is noticed that this control structure has been used in [37]. The input and output membership functions of the fuzzy system and its fuzzy rules are obtained from [37] and are shown in Figure 3 and Table 2, respectively.

In the industrial implementation and simulation, fractional order of $s$ is approximated with a transfer function of 
integer order. The most popular approximation for $s^{\alpha}$, in the frequency bound $\left[\omega_{L}, \omega_{H}\right]$, is a filter of the order of $2 N+1[38]:$

$$
s^{\alpha}=\omega_{H}^{\alpha} \prod_{k=-N}^{N} \frac{s-z_{k}}{s-p_{k}}, 0<\alpha<1
$$

where $z_{k}=-\omega_{L}\left(\omega_{H} / \omega_{L}\right)^{\frac{2 k+2 N+1+\alpha}{2(2 N+1)}}$, and $p_{k}=-\omega_{L}\left(\omega_{H} / \omega_{L}\right)^{\frac{2 k+2 N+1-\alpha}{2(2 N+1)}}$.

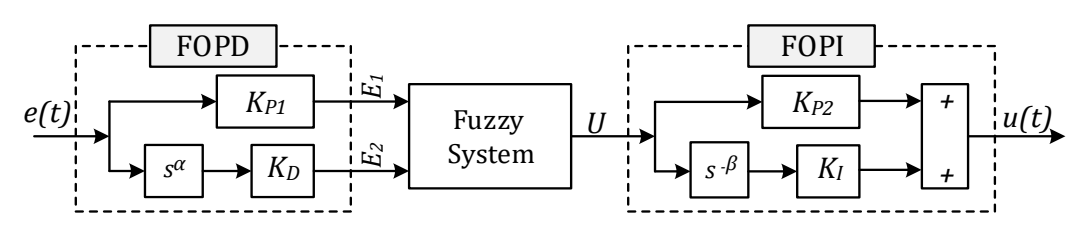

Figure 2: Combination of FOPD and Fuzzy Pre-compensated FOPI

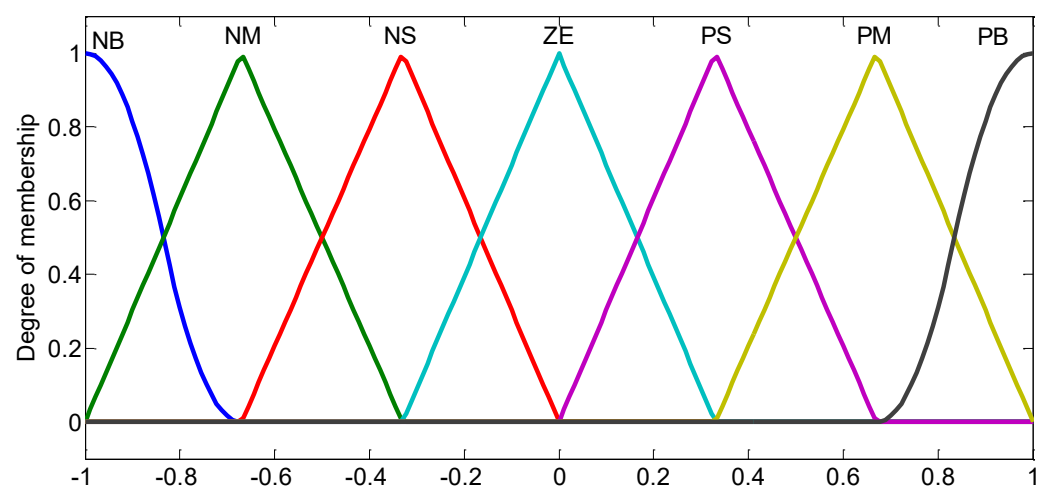

Figure 3: Membership Functions of Controller's Fuzzy System [37]

TABle 2. RulES OF CONTROLLER's FuZZy SYSTEM [37]

\begin{tabular}{c|c|c|c|c|c|c|c}
\hline $\begin{array}{c}\text { E2 } \rightarrow \\
\text { E1 }\end{array}$ & NL & NM & NS & ZR & PS & PM & PL \\
\hline \hline PL & ZR & PS & PM & PL & PL & PL & PL \\
\hline PM & NS & ZR & PS & PM & PL & PL & PL \\
\hline PS & NM & NS & ZR & PS & PM & PL & PL \\
\hline ZR & NL & NM & NS & ZR & PS & PM & PL \\
\hline NS & NL & NL & NM & NS & ZR & PS & PM \\
\hline NM & NL & NL & NL & NM & NS & ZR & PS \\
\hline NL & NL & NL & NL & NL & NM & NS & ZR \\
\hline
\end{tabular}

Design parameters of this controller are proportional gain $\left(K_{P 1}\right)$, integral gain $\left(K_{I}\right)$, derivative gain $\left(K_{D}\right)$, integral order $(\beta)$, and derivative order $(\alpha)$. The value of these parameters affects substantially the quality of the system response. In order to achieve the desired performance, fuzzy DE is used. Figure 4 shows the flowchart of fuzzy DE.

Fuzzy DE algorithm has been introduced in [34]. In fuzzy DE algorithm, the initial population consists of $n$ random vectors which include $d$ decision variables. The ith member of the population set in the Gth generation, $P_{i}^{G}$, is considered as:

$$
P_{i}^{G}=\left[\begin{array}{cccc}
p_{i, 1}^{G} & p_{i, 2}^{G} & \cdots & p_{i, d}^{G}
\end{array}\right], i=1,2, \cdots, n
$$

In each generation, the population of the next generation is produced using three operators of fuzzy mutation, crossover, and selection. In mutation for each $P_{i}^{G}$, three random vectors $P_{r_{1}}^{G}, P_{r_{2}}^{G}$, and $P_{r_{3}}^{G}$, are chosen and 


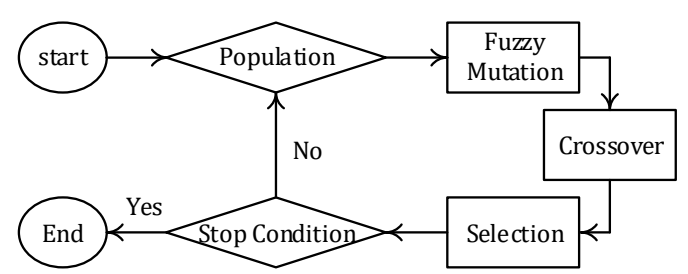

Figure 4: Fuzzy DE Flowchart

mutation vector, $M_{i}^{G}$, is produced as follows:

$$
M_{i}^{G}=P_{r_{1}}^{G}+F\left(P_{r_{2}}^{G}-P_{r_{3}}^{G}\right), r_{1} \neq r_{2} \neq r_{3} \neq i
$$

where $F$ is the mutation factor.

Crossover operation combines mutation vector, $M_{i}^{G}$, and parent vector, $P_{i}^{G}$, in order to constitute the polar vector, $Z_{i}^{G}$, as follows:

$$
z_{j, i}^{G}=\left\{\begin{array}{cc}
m_{j, i}^{G} & \text { if } r_{i} \leq C_{r} \text { or } j=J_{r} \\
p_{j, i}^{G} & \text { otherwise }
\end{array}, j=1,2, \ldots, d\right.
$$

where $r_{i}$ is a random variable between 0 and 1 and $J_{r}$ ensures $M_{i}^{G} \neq P_{i}^{G}$. The section operator chooses the best vector between the parent vector and test vector as:

$$
P_{i}^{G+1}=\left\{\begin{array}{lc}
Z_{i}^{G} & \text { if } f\left(U_{i}^{G}\right) \leq f\left(X_{i}^{G}\right) \\
P_{i}^{G} & \text { otherwise }
\end{array}\right.
$$

Two points should be considered for choosing $F$. First, at the primary iterations of the DE algorithm, a big value should be assigned to $F$ to speed up the exploration. By increasing the iterations, the smaller values should be assigned to $F$ for speeding up the exploitation. Second, the less is the relative distance of the population members, the less is the effective $F$ and vice versa. Population diversity is defined as:

$$
\operatorname{diversity}(G)=\frac{\sum_{a=1}^{n-1} \sum_{b=a+1}^{n}\left|\frac{P_{a}^{G}-P_{b}^{G}}{U-L}\right|}{2 d(n-1) n}
$$

where, $L$ and $U$ are the vectors containing the lower and upper bound of the population members. The above equation evaluates the average normal distance between the population members. In this regards, this criterion is effective for representing population diversity. For improving DE performance, fuzzy logic is used to determine $\mathrm{F}$ based on the number of generation and population diversity. The related membership function and fuzzy rules are shown in Figure 6 and Table 3, respectively. The Block diagram of the controlled microgrid with FOPD+FFOPI controller based on fuzzy DE algorithm is shown in figure 5.

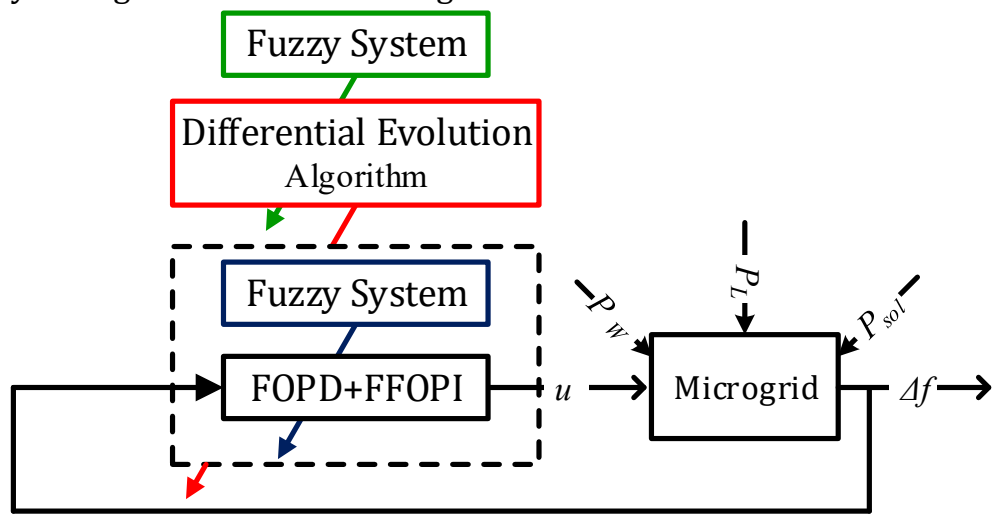

Figure 5: Block diagram of the microgrid by the proposed control approach 


\section{SIMULATION AND RESULTS}

In the load-frequency control problem, minimization of the frequency deviations is desired. On the other hand, control efforts are limited. The objective function of the optimization is the mean absolute of frequency deviations defined as follows:

$J=\frac{\int_{T_{1}}^{T}|\Delta f| d t}{T-T_{1}}$

The closed form of the optimization problem is as follows:

$\begin{array}{cc}\text { Min } & J(X) \\ \text { s.t. } & \left\{\begin{array}{c}L \leq X \leq U \\ |u| \leq u_{\text {max }}\end{array}\right.\end{array}$

Vector $X$ consists of the following decision variables:

$X=\left[\begin{array}{llllll}K_{p 1} & K_{p 2} & K_{I} & K_{D} & \beta & \alpha\end{array}\right]$

The uncontrolled inputs, wind power, solar thermal power, and variable load, are generated using the models of [36]. The Parameters of the system are similar to that of [35] and [36] and are shown in Table 4.

In this chapter, the parameters of equation (2) are chosen as $\omega_{H}=100, N=3$, and $\omega_{L}=0.01$.
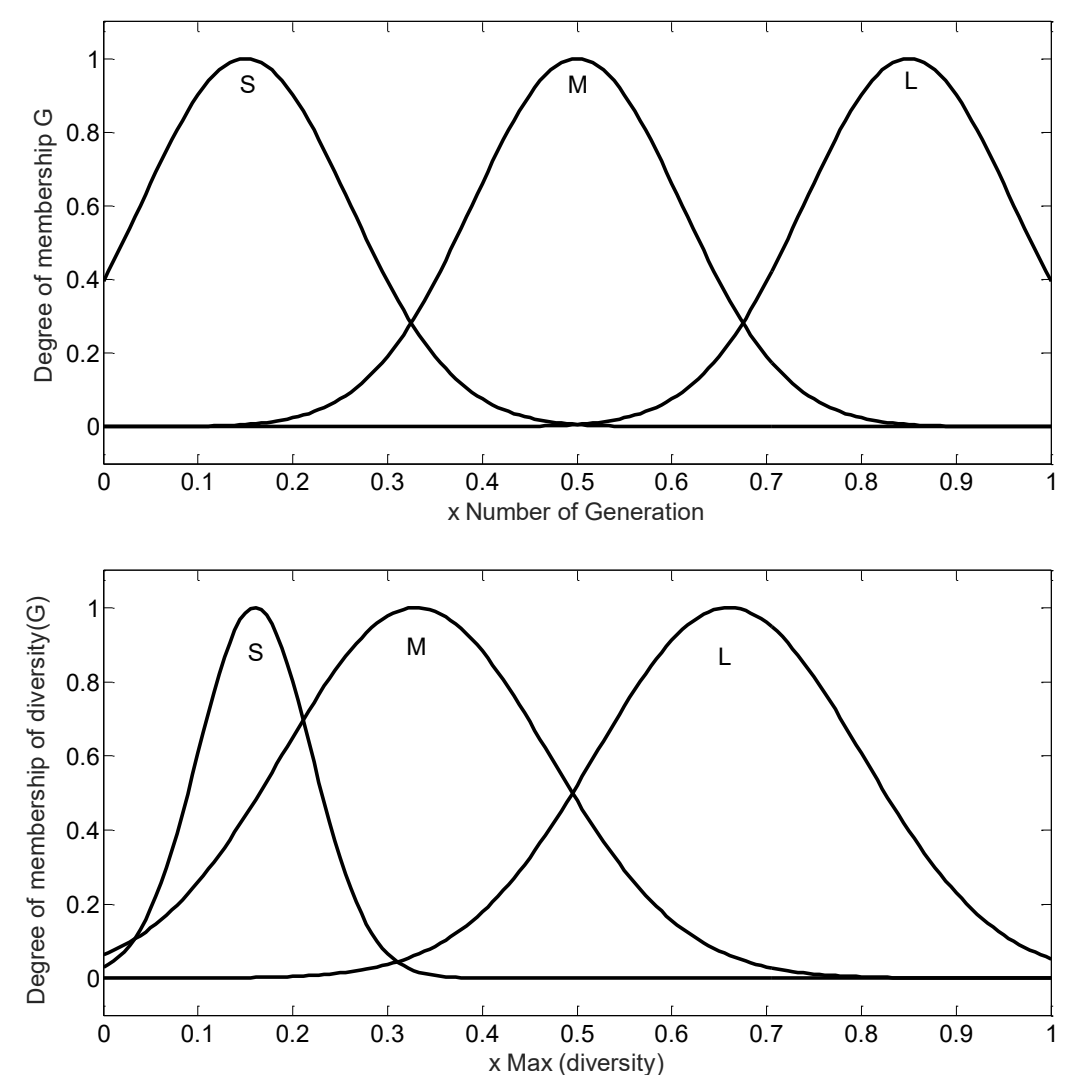


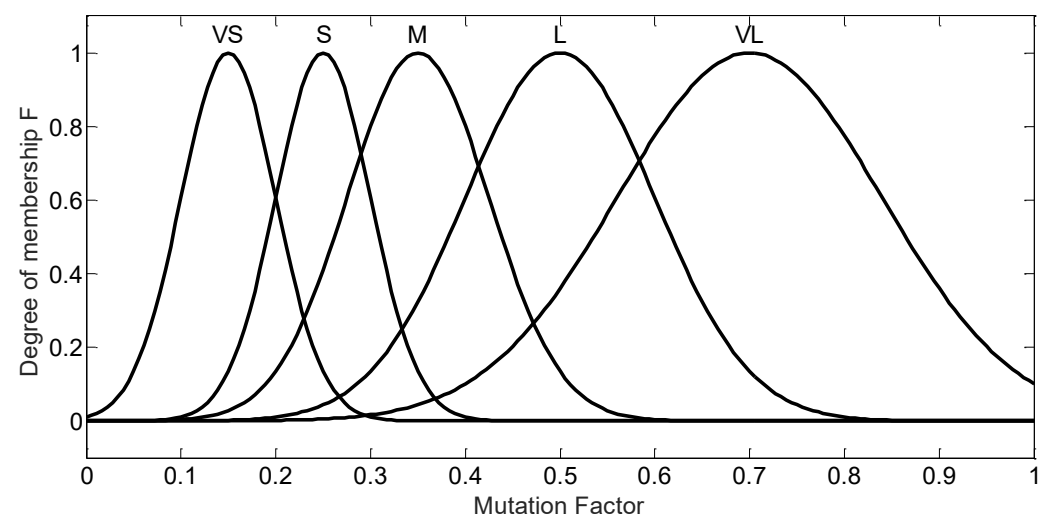

Figure 6: The Membership Functions of Fuzzy DE [34]

TABle 3. The Fuzzy RulES of FuZzy DE [34]

\begin{tabular}{c|c|c|c|c}
\hline \multicolumn{2}{c|}{} & \multicolumn{3}{|c}{$\operatorname{diversity}(G)$} \\
\cline { 3 - 5 } \multicolumn{2}{c|}{} & S & M & L \\
\hline \hline \multirow{2}{*}{$G$} & S & M & L & VL \\
\cline { 2 - 5 } & M & S & M & L \\
\cline { 2 - 5 } & L & VS & S & M \\
\hline
\end{tabular}

TABLE 4. The PARAMETER VALues OF THe TEST MiCROGRID

\begin{tabular}{|c|c|c|c|}
\hline Parameter & Value & Parameter & Value \\
\hline $\mathrm{K}_{\mathrm{w}}$ & 1 & R & 3 \\
\hline $\mathrm{K}_{\mathrm{FESS}}=\mathrm{K}_{\mathrm{BESS}}$ & 1 & $\mathrm{~T}_{\mathrm{w}}$ & $1.5 \mathrm{~s}$ \\
\hline $\mathrm{T}_{\mathrm{FESS}}=\mathrm{T}_{\mathrm{BESS}}$ & $0.1 \mathrm{~s}$ & $\mathrm{~T}_{\text {IN }}$ & $0.04 \mathrm{~s}$ \\
\hline $\operatorname{Max}\left(P_{\text {FESS }}\right)=\operatorname{Max}\left(P_{\text {BESS }}\right)$ & 0.11 & $\mathrm{~T}_{\text {IC }}$ & $0.004 \mathrm{~s}$ \\
\hline $\operatorname{Max}(\mathrm{PFC})$ & 0.48 & $\mathrm{~K}_{\mathrm{FC}}$ & 1 \\
\hline $\operatorname{Max}\left(\mathrm{P}_{\mathrm{DEG}}\right)$ & 0.45 & $\mathrm{~T}_{\mathrm{FC}}$ & 0.26 \\
\hline $\operatorname{Max}\left(\mathrm{P}_{\text {FESS }}^{\prime}\right)=\operatorname{Max}\left(\mathrm{P}^{\prime}{ }_{\text {BESS }}\right)$ & 0.05 & $\mathrm{~T}_{\mathrm{G}}$ & 0.08 \\
\hline $\operatorname{Max}\left(\mathrm{P}_{\mathrm{FC}}^{\prime}\right)$ & 1 & $\mathrm{~T}_{\mathrm{T}}$ & 0.4 \\
\hline $\operatorname{Max}\left(\mathrm{P}^{\prime}{ }_{\mathrm{DEG}}\right)$ & 0.5 & $\mathrm{D}$ & 0.015 \\
\hline$\Gamma_{\text {LOAD }}$ & $\begin{array}{c}0.9 \mathrm{u}(\mathrm{t})+0.03 \mathrm{u}(\mathrm{t}-110)+0.03 \mathrm{u}(\mathrm{t}- \\
130)+0.03 \mathrm{u}(\mathrm{t}-150)+0.15 \mathrm{u}(\mathrm{t}- \\
170)+0.1 \mathrm{u}(\mathrm{t}-190)\end{array}$ & $\mathrm{H}$ & $1 / 12$ \\
\hline$\Gamma_{\text {load }}$ & $0.02 \mathrm{u}(\mathrm{t})$ & $\eta_{\text {load }}$ & 0.9 \\
\hline $\mathrm{G}_{\text {load}(s)}$ & $1-300 /(300 s+1)-1 /(1800 s+1)$ & Bload & 10 \\
\hline$\Gamma_{\text {wind }}$ & $0.24 \mathrm{u}(\mathrm{t})-0.04 \mathrm{u}(\mathrm{t}-140)$ & $\eta_{\text {wind }}$ & 0.8 \\
\hline $\mathrm{G}_{\text {wind }}(\mathrm{s})$ & $10^{4} s /\left(10^{4} s+1\right)$ & $\beta_{\text {wind }}$ & 10 \\
\hline$\Gamma_{\text {sol }}$ & $0.05 u(t)-0.02 u(t-180)$ & $\eta_{\text {sol }}$ & 0.1 \\
\hline $\mathrm{G}_{\mathrm{sol}}(\mathrm{s})$ & $10^{4} \mathrm{~s} /\left(10^{4} \mathrm{~s}+1\right)$ & $\mathrm{B}_{\text {sol }}$ & 10 \\
\hline
\end{tabular}

For the microgrid of Figure 1, PID controller $\left(\frac{U(s)}{\Delta F(s)}=K_{P}+\frac{K_{I}}{s}+K_{D} s\right)$, FOPID controller ( $\frac{U(s)}{\Delta F(s)}=K_{P}+\frac{K_{I}}{s^{\beta}}+K_{D} s^{\alpha}$ ), and a combination of FOPD and Fuzzy Pre-compensated FOPI controller of Figure 2 are designed and the related parameters of them are optimized using fuzzy DE. In this regards, these controllers are named FDE-PID, FDE-FOPID, and FDE-FOPD+FFOPI, respectively. Also, FOPD+FFOPI controller is designed, and its parameters are optimized using DE. This controller is named DE-FOPD+FFOPI. In Figure 7, the frequency deviations of the microgrid after implementation of the designed controllers are shown and compared.

In all optimization algorithms, the same number of iterations is considered as the terminal condition. The controllers are sorted based on the value of the oscillation frequency of the frequency deviations as follows: FDEPID, DE-FOPD-FFOPI, FDE-FOPD-FFOPI, and FDE-FOPID controller. In this list, FDE-PID has the highest and FDEFOPID has the least oscillation frequency. Negative frequency variations indicate a decrease in frequency and positive indicate an increase in frequency. The maximum values of increase and decrease of frequency variations are observed with FDE-FOPID, DE-FOPD-FFOPI, FDE-FOPD-FFOPI, and FDE-PID controllers, respectively. The 
FDE-FOPID controller has the lowest oscillation frequency and the highest frequency reduction, while the FDEPID controller has the highest oscillation frequency and the lowest frequency reduction. In other words, the improvement of the oscillation frequency of frequency deviations is inconsistent with its improvement of the maximum increase and decrease. It can be concluded that the proposed controller has created a favorable tradeoff between the oscillation frequency and the maximum value of increasing and decreasing of the frequency variations.

In Figure 8, $\log 10$ of the values of the objective function in the sequential iterations is compared for DEFOPD+FFOPI and FDE-FOPD+FFOPI. In order to compare DE and fuzzy DE in determining the parameters of FOPDFFOPI controller rationality and fairly, the random initial population of them are selected identical and their terminal condition is chosen the same number of iterations. As it is expected by applying the fuzzy system, at the primary iterations of the DE algorithm, the exploration speeds up and at the last iterations, the exploitation speeds up. In other words, the fuzzy system improves the performance of the DE algorithm from the viewpoint of convergence rate and more precise searching, considerably.

In Table 5, the performance of the controllers are more precisely compared with quantitative indices $J, \max (|u|)$ , and $\max (|\Delta f|)$. It is observed that the mean absolute of frequency deviations of the FDE-FOPID controller is less than that of FDE-PID and the performance of DE-FOPD+FFOPI in decreasing the mean absolute of frequency deviations is better than that of FDE-PID controller. The results show that by using the FDE-FOPID controller there is a good trade-off between these three quantitative and conflict indices. This demonstrates the capability of the fractional calculus. There is no logical relationship between $\max (|u|)$ and the other two indices but the constraint of limited amplitude of the control effort is satisfied. The least mean absolute of frequency deviations is pertaining to the FDE-FOPD+FFOPI controller. These results confirm the superiority of the proposed control method with the fuzzy DE optimization method.

Table 5. Quantitative Comparison of the Performance with Designed Controllers

\begin{tabular}{c|c|c|c}
\hline $\begin{array}{c}\text { Index } \rightarrow \\
\text { Controller } \downarrow\end{array}$ & $\begin{array}{c}J \\
\times 10^{-3}\end{array}$ & $\max (|u|)$ & $\begin{array}{c}\max (|\Delta f|) \\
\times 10^{-2}\end{array}$ \\
\hline \hline FDE-PID & 4.8414 & 30.218 & 3.7857 \\
\hline FDE-FOPID & 2.489 & 7.5941 & 7.395 \\
\hline DE-FOPD+FFOPI & 1.9617 & 70.797 & 7.324 \\
\hline FDE-FOPD+FFOPI & 1.6097 & 38.665 & 4.7752 \\
\hline
\end{tabular}

It is noteworthy that considering the variations of the system parameters is essential when evaluating the performance of a load-frequency control system. Ignoring the parameter uncertainties can, in practice, lead to undesirable system behavior and the failure in achieving the desired control objectives. In this regard, the uncertainty of the parameters D, H, R, TFC, TG, and TT are considered and the frequency deviations of the microgrid are assessed under the variation of them for evaluating the robustness of the proposed approach. The related results are shown in Figures 9 and 10 and Table 6. The results confirm the robustness of the proposed approach.

As shown in Figures 9 and 10, in spite of the substantial change in the parameters of the system, frequency deviations are retained in an acceptable range and the control performance is still acceptable. The quantitative results in Table 6 demonstrate that variation of the parameters affects the maximum of the frequency variation $(\max (|\Delta f|))$ more than J. By increasing the parameters, J does not change dramatically and the maximum of the frequency variation changes more. However, by decreasing the parameters both indices, J and $\max (|\Delta f|)$, change. It is also observed that by decreasing $\mathrm{H}$ and increasing $\mathrm{TG}, \max (|\Delta f|)$ is maximized. 

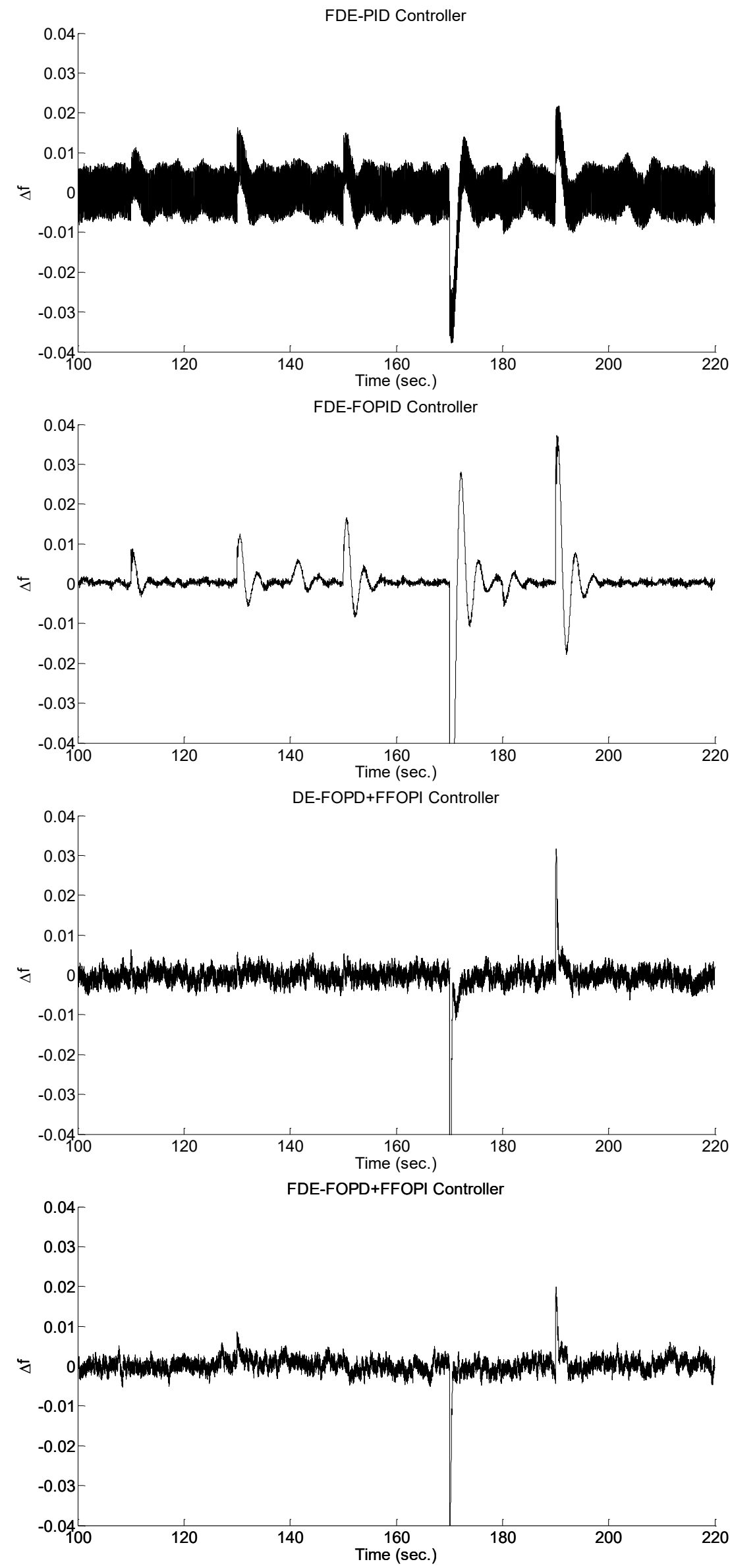

Figure 7 Comparison of Frequency Deviations with Designed Controllers 


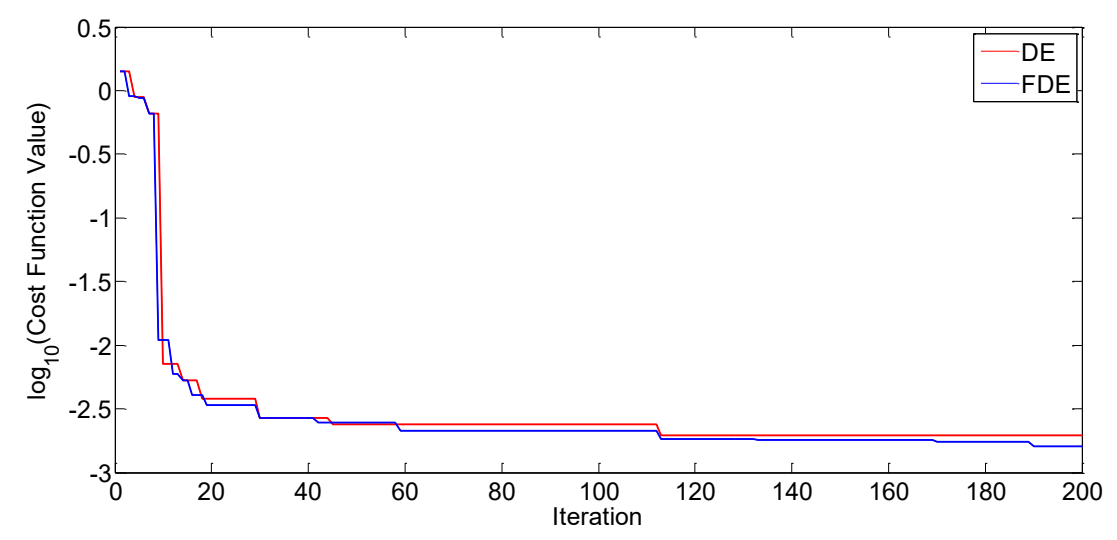

Figure 8 Comparison of the Objective Function of Fuzzy DE and DE
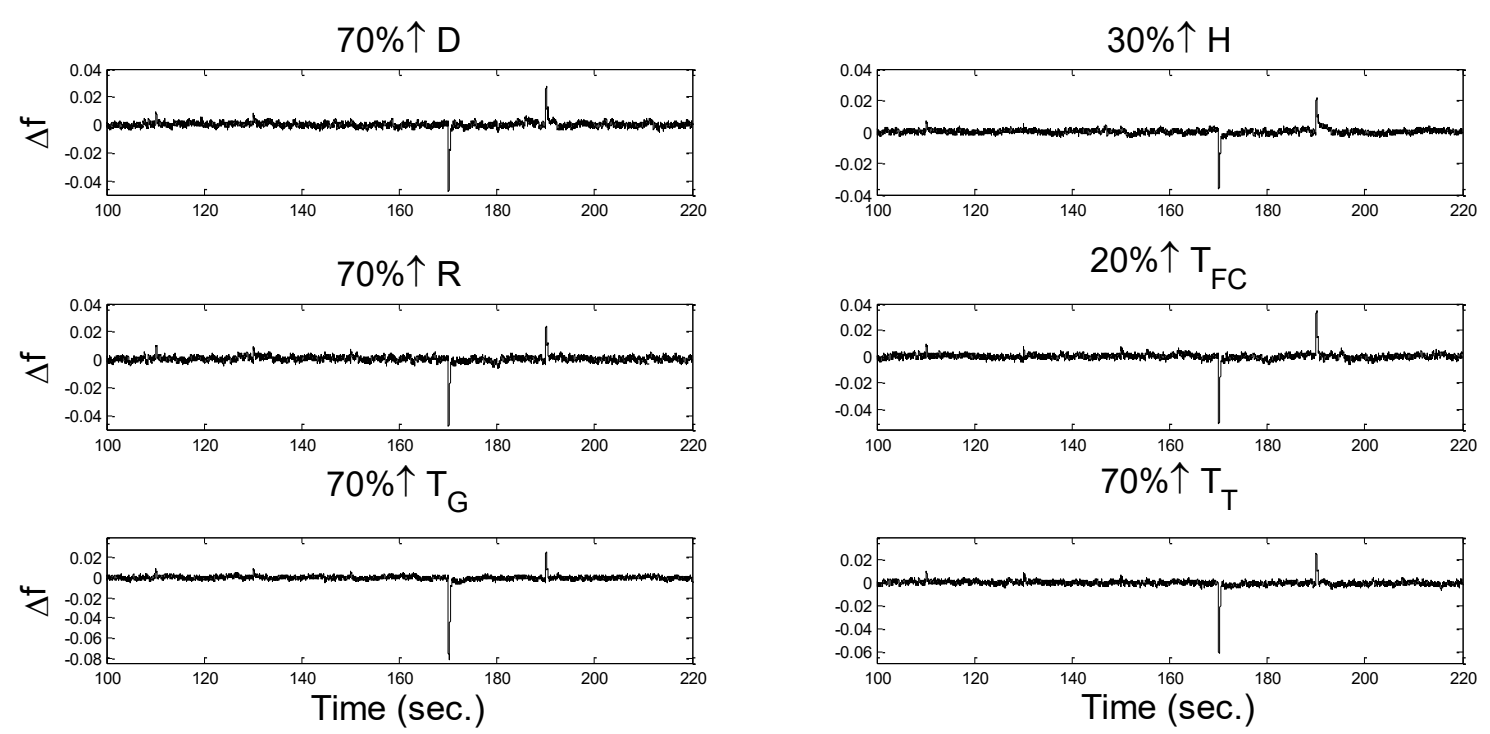

Figure 9 Robust Performance Under Increasing The Parameters With FDE-FOPD+FFOPI.
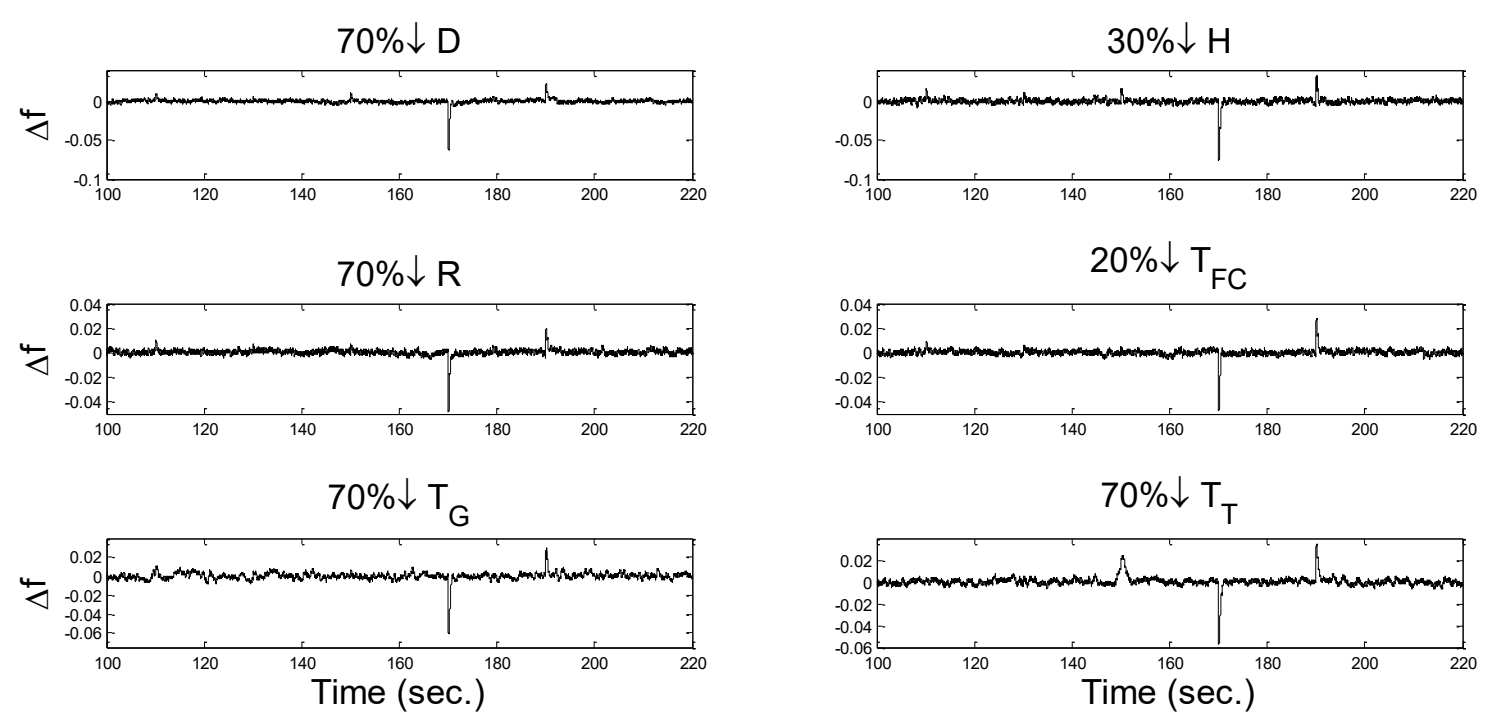

Figure 10 Robustness Performance Under Decreasing The Parameters With FDE-FOPd+FFoPI controller. 
Table 6. Robust Performance Under Variation The Parameters With FDE-FOPD+FFOPI.

\begin{tabular}{|c|c|c|c|c|}
\hline & $\begin{array}{c}J \\
\times 10^{-3}\end{array}$ & $\begin{array}{c}\max (|\Delta f|) \\
\times 10^{-2}\end{array}$ & $\begin{array}{c}J \\
\times 10^{-3}\end{array}$ & $\begin{array}{c}\max (|\Delta f|) \\
\times 10^{-2}\end{array}$ \\
\hline & \multicolumn{2}{|c|}{ Increase } & \multicolumn{2}{|c|}{ Decrease } \\
\hline $70 \% \mathrm{D}$ & 1.5808 & 4.7672 & 1.7043 & 6.2765 \\
\hline $30 \% \mathrm{H}$ & 1.2551 & 3.6343 & 2.3250 & 7.6134 \\
\hline $70 \% \mathrm{R}$ & 1.6785 & 4.7939 & 1.6312 & 4.8079 \\
\hline $20 \% \mathrm{~T}_{\mathrm{FC}}$ & 1.6333 & 5.0586 & 1.6194 & 4.7472 \\
\hline $70 \% \mathrm{~T}_{\mathrm{G}}$ & 1.6867 & 8.2170 & 2.5443 & 6.1443 \\
\hline $70 \% \mathrm{~T}_{\mathrm{T}}$ & 1.6060 & 6.1088 & 2.3178 & 5.7546 \\
\hline
\end{tabular}

\section{CONCLUSION}

In this chapter, an optimal combination of FOPD and fuzzy pre-compensated FOPI approach was designed for reducing the frequency deviations of an islanded microgrid. Nonlinear phenomena such as saturation and ratelimiting power have been added to the linear model of the microgrid components. The dynamic model of various perturbations, including load changes, changes in wind speed and changes in sunlight, were considered. The fractional order structure was proposed because of increasing the controller degrees of freedom and its robust performance. The parameters of the controller were determined via differential evolution (DE) algorithm which was improved by the fuzzy system. In the proposed optimization approach, the objective function was to optimize the average magnitude of the frequency variations while the control effort amplitude does not exceed the predetermined value. Control effort has been limited by including a relevant constraint in the optimization model. Simulations were performed using MATLAB, and the performance of the proposed control approach was compared with the performance of Fractional order PID and PID controllers whose parameters were determined by fuzzy differential evolution algorithm. Frequency deviations had the smallest magnitude with PID controller and it had the least frequency of variations with FOPID controller. Using FDE-FPD-FFOPI controller, a good tradeoff was made between the two conflict criteria: variation's frequency and magnitude of frequency deviations. The same number of iterations was chosen as the Terminal condition of the fuzzy differential evolution algorithm to determine the parameters of all three controllers. The simulation results showed improvement of frequency deviations via implementing the proposed control schema. To illustrate the superior performance of the fuzzy differential evolution algorithm compared to the differential evolution algorithm, the FPD-FFOPI controller parameters were determined using both algorithms. To fair and rational comparison, the initial population was selected random and identical and the same number of iterations was chosen as the Terminal condition , In both optimization. Applying the fuzzy system, at the primary iterations of the DE algorithm, the exploration and at the last ones, the exploitation speeded up. In another word, the fuzzy system improves the performance of the DE algorithm from the viewpoint of convergence rate and more precise searching, considerably.

For quantitative analysis of simulation results, the maximum amplitude of the control effort and frequency variations were calculated. Since it is important to evaluate the performance of the load frequency control system under the parameter uncertainties, The performance of the proposed controller is investigated by considering significant changes in the parameters T, D, H, R, TFC, TG, and TT. The quantitative and qualitative results of this investigation showed that despite considerable variations in system parameters, the frequency variations remained within the acceptable range. A performed sensitivity analysis confirmed the robustness of the proposed approach.

\section{REFERENCES}

[1] MR Salehizadeh, A Rahimi-Kian, and K Hausken. "A leader-follower game on congestion management in power systems." Game Theoretic Analysis of Congestion, Safety and Security. Springer, Cham, pp. 81-112, 2015.

[2] MR Salehizadeh, A Rahimi-Kian, and M Oloomi-Buygi. "A multi-attribute congestion-driven approach for evaluation of power generation plans." International Transactions on Electrical Energy Systems, Vol. 25, No. 3, pp. 482-497, 2015.

[3] Ma, Jia-Jun, Gang Du, and Bai-Chen Xie. "CO2 emission changes of China's power generation system: Input-output subsystem analysis." Energy Policy, No. 124, pp. 1-12, 2019.

[4] Goldthau, Andreas, and Nick Sitter. "Regulatory or market power Europe? EU leadership models for international energy governa nce." New Political Economy of Energy in Europe. Palgrave Macmillan, Cham, pp. 27-47, 2019.

[5] M Oloomi-Buygi, and MR Salehizadeh. "Toward fairness in transmission loss allocation." 2007 Australasian Universities Power Engineering Conference. IEEE, 2007.

[6] P. K. Ray, S. R. Mohanty and N. Kishor, "Proportional-integral controller based small-signal analysis of hybrid distributed generation systems" Energy Conversion and Management, Vol. 52, No. 4, pp. 1943-1954, 2011.

[7] Dhanalakshmi, R \& Palaniswami, S. "Load frequency control of wind diesel hydro hybrid power system using conventional PI controller". Vol. 60. pp. 630-641, 2011.

[8] Prakash K. Ray, Soumya R. Mohanty, Nand Kishor, "Proportional-integral controller based small-signal analysis of hybrid distributed 
generation systems" Energy Conversion and Management, Vol. 52, No. 4, pp. 1943-1954, 2011.

[9] G. Shankar and V. Mukherjee, "Load frequency control of an autonomous hybrid power system by quasi-oppositional harmony search algorithm" Int. Journal of Electrical Power \& Energy Systems, Vol. 78, pp. 715-734, 2016.

[10] M. A. El-Hameed and A. A. El-Fergany, "Efficient frequency controllers for autonomous two-area hybrid microgrid system using socialspider optimiser" IET Generation, Transmission Distribution, Vol. 11, No. 3, pp. 637-648, 2017.

[11] S. K. Pandey, S. R. Mohanty, N. Kishor and J. P. S. Cataleo, "Frequency regulation in hybrid power systems using particle swarm optimization and linear matrix inequalities based robust controller design" Int. Journal of Electrical Power \& Energy Systems, Vol. 63, pp. 887-900, 2014.

[12] H. Bevrani, F. Habibi, P. Babahajyani, M. Watanabe and Y. Mitani, "Intelligent Frequency Control in an AC Microgrid: Online PSO-Based Fuzzy Tuning Approach" IEEE Trans. on Smart Grid, Vol. 3, No. 4, pp. 1935-1944, 2012.

[13] F. Jamshidi, S. L. Emamzadehei and M. M. Ghanbarian, "Using Fuzzy PI controller Optimized by PSO for Frequency Control of sland Microgrids" Journal of soft Computing and Information technology, Vol. 6, No. 1, pp. 36-43, 2017.

[14] H. Shayeghi and A. Ghasemi, "Improvement of Frequency Fluctuations in Microgrids Using an Optimized Fuzzy P-PID Controller by Modified Multi Objective Gravitational Search Algorithm" Iranian Journal of Electrical and Electronic Engineering, Vol. 12, N o. 4, pp. 241256, 2016.

[15] A. Parisioa, E. Rikos, G. Tzamalis and L. Glielmo, “Use of model predictive control for experimental microgrid optimization," Applied Energy, Vol. 115, No. 15, pp. 37-46, 2014.

[16] S. R. Cominesi, M. Farina, L. Giulioni, B. Picasso and R. Scattolini, "A Two-Layer Stochastic Model Predictive Control Scheme for Microgrids" IEEE Transactions on Control Systems Technology, Vol. 26, No. 1, pp. 1-13, 2018.

[17] J. Pahasa and I. Ngamroo, "PHEVs bidirectional charging/ discharging and soc control for microgrid frequency stabilization using multiple MPC” IEEE Trans. on Smart Grid, Vol. 6, No. 2, pp. 526-533, 2015.

[18] J. Pahasa and I. Ngamroo, "Coordinated Control of Wind Turbine Blade Pitch Angle and PHEVs Using MPCs for Load Frequency Control of Microgrid" IEEE Systems Journal, vol. 10, no. 1, pp. 97-105, 2016.

[19] C. Wang, X. Li, L. Guo and Y. W. Li, "A Nonlinear-Disturbance-Observer-Based DC-Bus Voltage Control for a Hybrid AC/DC Microgrid," in IEEE Transactions on Power Electronics, Vol. 29, No. 11, pp. 6162-6177, Nov. 2014.

[20] H. R. Baghaee, M. Mirsalim and G. B. Gharehpetian, "Performance Improvement of Multi-DER Microgrid for Small- and Large-Signal Disturbances and Nonlinear Loads: Novel Complementary Control Loop and Fuzzy Controller in a Hierarchical Droop-Based Control Scheme," in IEEE Systems Journal, Vol. 12, No. 1, pp. 444-451, March 2018.

[21] Chaoxu Mu, Weiqiang Liu, Wei Xu, and Md. Rabiul Islam, "Observer-Based Load Frequency Control for Island Microgrid with Photovoltaic Power," International Journal of Photoenergy, Vol. 2017, Article ID 2851436, 11 pages, 2017. https://doi.org/10.1155/2017/2851436.

[22] Yanmei Tang, Yan Bai, Congzhi Huang, Bin Du, "Linear active disturbance rejection-based load frequency control concerning high penetration of wind energy," Energy Conversion and Management, Vol. 95, pp. 259-271, 2015.

[23] Adel Ahmadi, Mohammad Aldeen, "Robust overlapping load frequency output feedback control of multi-area interconnected power systems," International Journal of Electrical Power \& Energy Systems, Vol. 89, pp. 156-172, 2017.

[24] H. Cai and G. Hu, "Distributed Nonlinear Hierarchical Control of AC Microgrid via Unreliable Communication, " in IEEE Transactions on Smart Grid, vol. 9, no. 4, pp. 2429-2441, July 2018.

[25] Vijay P. Singh, Soumya R. Mohanty, Nand Kishor, Prakash K. Ray, "Robust H-infinity load frequency control in hybrid distributed generation system," International Journal of Electrical Power \& Energy Systems, Vol. 46, pp. 294-305, 2015

[26] I. Pan and S. Das, "Fractional Order AGC for Distributed Energy Resources Using Robust Optimization," in IEEE Transactions on Smart Grid, vol. 7, no. 5, pp. 2175-2186, Sept. 2016.

[27] Indranil Pan, Saptarshi Das, "Fractional order fuzzy control of hybrid power system with renewable generation using chaotic PSO," ISA Transactions, Vol. 62, pp. 19-29, 2016.

[28] Mohammad-Hassan Khooban, Taher Niknam, Frede Blaabjerg, Pooya Davari, Tomislav Dragicevic, "A robust adaptive load frequency control for micro-grids," ISA Transactions, Vol. 65, pp. 220-229, 2016.

[29] Mohammad Hassan Khooban, Taher Niknam, Frede Blaabjerg, Tomislav Dragičević, "A new load frequency control strategy for microgrids with considering electrical vehicles," Electric Power Systems Research, Vol. 143, pp. 585-598, 2017.

[30] Y. Hain, R. Kulessky and G. Nudelman, "Identification-based power unit model for load-frequency control purposes," in EEE Transactions on Power Systems, vol. 15, no. 4, pp. 1313-1321, Nov. 2000.

[31] Yang Mi, Yang Fu, Dongdong Li, Chengshan Wang, Poh Chiang Loh, Peng Wang, "The sliding mode load frequency control for hybrid power system based on disturbance observer," International Journal of Electrical Power \& Energy Systems, Vol. 74, pp. 446-452, 2016.

[32] İlhan Kocaarslan, Ertuğrul Çam, "Fuzzy logic controller in interconnected electrical power systems for load-frequency control," International Journal of Electrical Power \& Energy Systems, Vol. 27, Issue 8, pp. 542-549, 2005.

[33] F. Jamshidi and M. M. Ghanbarian , "Robust Frequency Control of Islanded Microgrids: ICA-Based FFOPID Control Approach" Computational Intelligence in Electrical Engineering, Vol. 8, No. 1, pp. 51-62, 2017.

[34] M. Salehpour, A. Jamali, A. Bagheri and N. Nariman-zadeh, "A new adaptive differential evolution optimization algorithm based on fuzzy inference system" Engineering Science and Technology, an International Journal, Vol. 20, No. 2, pp. 587-597, 2017.

[35] H. Wang, G. Zeng, Y. Dai, D. Bi, J. Sun and X. Xie, “Design of a Fractional Order Frequency PID Controller for an Islanded Microgrid: A MultiObjective Extremal Optimization Method" Energies, Vol. 10, No. 1502, pp. 1-18, 2017.

[36] D. C. Das, A. Roy and N. Sinha, "GA based frequency controller for solar thermal-diesel-wind hybrid energy generation/energy storage system" Electrical Power and Energy Systems, Vol. 43, pp. 262-279, 2012.

[37] S. Das, I. Pa, S. Das, \& A. Gupta. "A novel fractional order fuzzy PID controller and its optimal time domain tuning based on integral performance indices." Engineering Applications of Artificial Intelligence, 25(2), 430-442, 2012.

[38] A. Oustaloup, F. Levron, B. Mathieu and F. M. Nanot, "Frequency-band complex noninteger differentiator: characterization and synthesis," in IEEE Transactions on Circuits and Systems I: Fundamental Theory and Applications, vol. 47, no. 1, pp. 25-39, Jan. 2000. 